\title{
Synthesis of New Oxazepine and Thaizolidine Compounds derived from Pyrimidine-2(1H)-one
}

\author{
Zainab H. Sulyman ${ }^{\text {; }}$; Natiq G. Ahmed ${ }^{2}$ \\ ${ }^{1,2}$ Department of Chemistry, College of Education for Pure Science, University of Mosul, Mosul, Iraq.
}

Email: ${ }^{1 *}$ sulymanzainab5@gmail.com, ${ }^{2}$ natiq.ahmed@yahoo.com

(Received July 10, 2019; Accepted October 14, 2019; Available online June 01, 2020)

DOI: 10.33899/edusj.2019.125908.1004, (c) 2020, College of Education for Pure Science, University of Mosul.

This is an open access article under the CC BY 4.0 license (http://creativecommons.org/licenses/by/4.0/).

\begin{abstract}
In this paper the compounds (11-20) (methyl pyrimidine - 2(1H)-one and others phenyl pyrimidine $-2(1 \mathrm{H})-$ one) that had been already prepared from $\alpha, \beta$ unsaturated carbonyl compounds that are called chalcones, these compounds are usually prepared from the reaction of different aldehydes (4-methoxy benzaldehyde, 2-nitro benzaldehyde, 3-nitrobenzaldehyde, 4-N,N-dimethyle amino benzaldehyde, benzaldehyde, 4-nitro benzaldehyde) with different ketones (acetone, acetophenone, 2-nitro acetophenone, 3-nitro acetophenone) After preparing and purifying them a suitable mesurment for physical, chemical and spectroscopic properties had been made to get chalcones that reacted with urea under known chemical conditions to get the pyrimidinone compounds that we need. Pyrimidine compounds had been reacted with two aromatic amines (2,4-dinitro aniline and 4- amino acetophenone) using glacial acetic acid as catalyst in absolute ethanol giving a new compounds of schiff's bases (21-40). New thiazolidine 4-one (41-50) had been synthesized from the reacting of Schiff's bases (21-30) with thioglycolic acid in absolute ethanol. 1,3oxazepine derivatives. (51-60) had been prepared from reaction between Schiff's bases (31-40) and malic anhydrid in absolut ethanol. The structures of the Synthesized compounds had been estimated by IR, ${ }^{1} \mathrm{H}-\mathrm{NMR}$ and some physical data.
\end{abstract}

Keywords: pyrimidine, Schiff's bases, Thiazolidine, 1,3- oxazepine
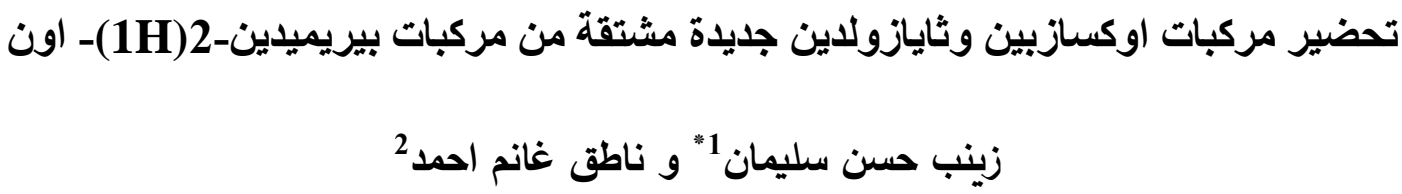

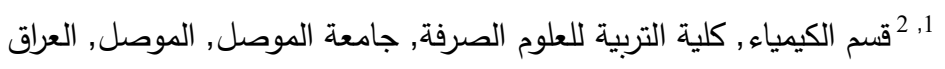

الخلاصة

في هذا البحث تم تحضـــير المركبات (20-11) (مثيل بريميدين-2(1H)- أون ومركبات اخرى من فنيل بريميدين -

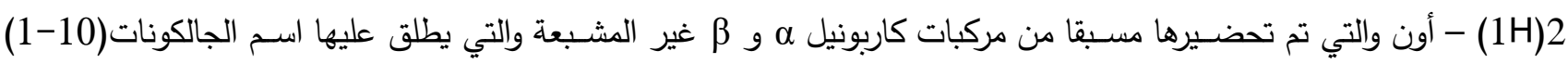

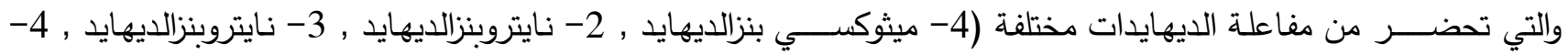

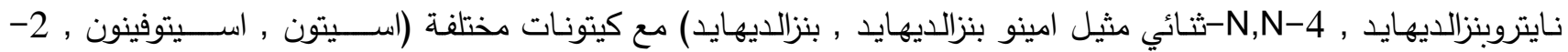




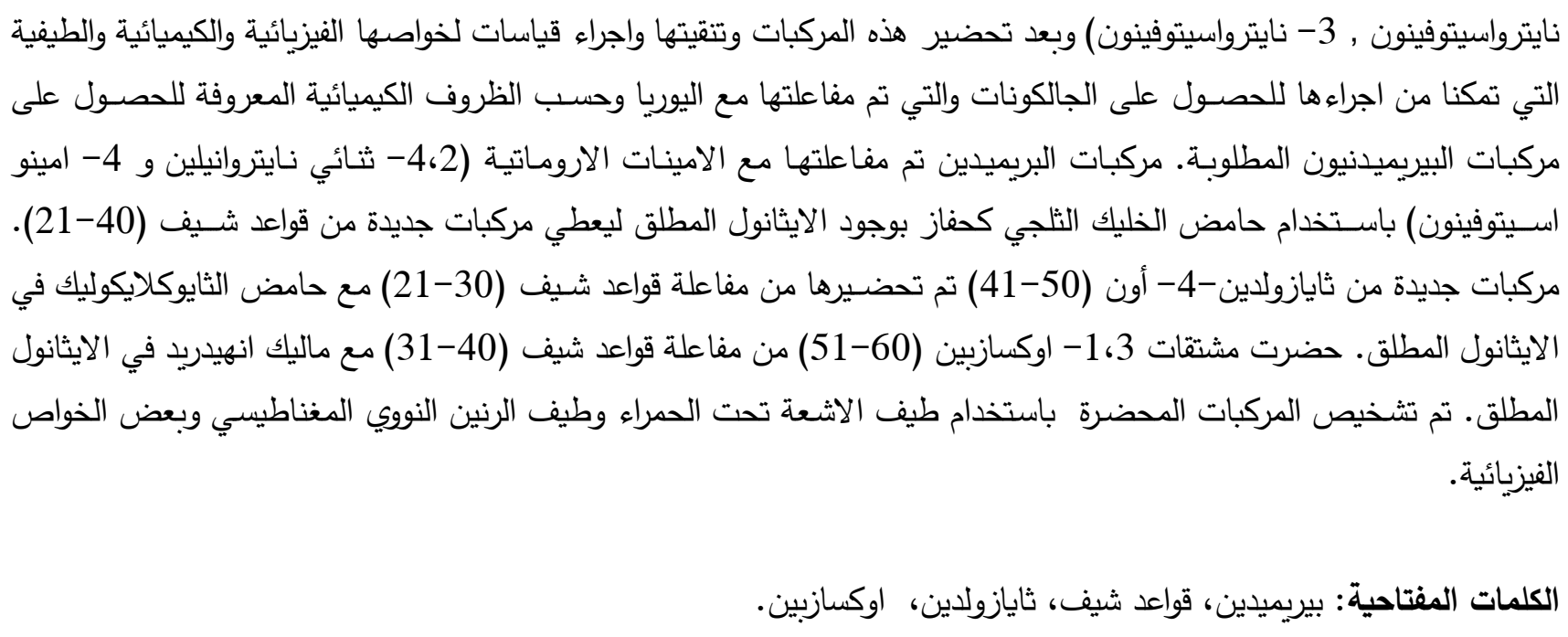

\section{INTRODUCTION}

Pyrimidines were used as substrates in the preparation of many schiff's bases which undergo ring closure (cyclo addition and replacement reaction) [1] to prepare thia- zolidine derivatives which have various pharmacological activities such as antibacterial [2], anti-fungal [3], anti-Cancer [4], anti con-vulsant [5] and herbicidal actions [6]. 1,3-oxazipene derivatives also Synthesized from the reaction of Schiff's bases with maleic anhydrid these compounds have been found to exhibit biological activites including anti-bacterial, antifungal [7,8], anticancer [9].

\section{EXPERIMENTAL}

All reagents and compounds are from Fluka and BDH. Melting points are measured using: Electro thermal IA 9100 melting points apparatus type (not corrected). FT-IR Spectra were recorded on BRUKER ALPHLA FTIR Germany. ${ }^{1} \mathrm{H}-\mathrm{NMR}$ spectra were recorded by NMREADY 60 PRO. Using DMSO-d6 as a solvent in college of education and science Ibn al-haitham in Baghdad/Iraq. Synthesis of chalcones (1-10) [10,11]

0.1 mole of benzaldehyde or one of its compensations dissolved in $10 \mathrm{ml}$ of EtOH was added to 0.1 mole of acetone or acetophenone or one of its compensations that had been also dissolved in $10 \mathrm{ml}$ of $\mathrm{EtOH}$, the mixture was stirred in aqueous alkali $(40 \% \mathrm{NaOH})$ in room temprature for 5 hours. Table (1) involves physical properties as reported in the literature[12]

\section{Synthesis of Pyrimidine-2(1H)-one (1-10) [13]:}

(0.01) mole of chalcones (1-10) had been dissolved in EtOH abs. This was added to (0.01) mole of urea which dissolved in $20 \mathrm{ml}$ of EtONa then reflux the mixture for 12 hours. The solvent had been concentrated and poured into ice cold water with constant stirring then it was neutralize with acid Solid produce filtered and washed with water. Table (2) involves the physical properties. Synthesis of Schiff's Bases (21-40) [14]:

(0.01) mole of pyrimidines compounds (11-20) was added to (0.01) mole of aromatic amine (2,4-dinitro aniline , 4- amino acetophenon in abs. EtOH (25) $\mathrm{ml}$ with some drops of glacial acetic acid is had been stirred for 4 hours . Table $(3,4)$ involves the physical properties. 


\section{Synthesis of Thiazolidine Derivatives (41-50) [15-17]:}

(0.001) mole of Schiff's bases (21-30) in abs. EtOH (15 ml) was added to (0.001) mole of thioglycolic acid, this mixture was refluxed for 5 hours, and treated with potassium bicarbonate to produce a compound, then product filtered. Table (5) includes the physical properties.

\section{Synthesis of 1,3- Oxazepine Derivatives (51-60)[18]:}

Mixture of Schiff's bases (31-40) (0.0004) mole was added to (0.0006 mole) of maleic anhydride in (20) $\mathrm{ml}$ of abs. Ethanol was refluxed for 6 hours. The product was filtered and washed with water. Table (6) involves the physical properties

Table (1)The Physical Properties of Compound (1-10)

\begin{tabular}{|c|c|c|c|c|c|c|c|}
\hline $\begin{array}{c}\text { Comp. } \\
\text { No. }\end{array}$ & $\mathbf{R}$ & $\mathbf{R}_{\mathbf{1}}$ & $\begin{array}{c}\text { Molecular } \\
\text { formula }\end{array}$ & $\begin{array}{c}\mathbf{M} . \\
\mathbf{W t}\end{array}$ & $\mathbf{m . p} \mathbf{c}$ & Colour & $\begin{array}{c}\text { Yiel } \\
\mathbf{d} \%\end{array}$ \\
\hline 1 & $4-\mathrm{OCH}_{3}$ & $\mathrm{CH}_{3}$ & $\mathrm{C}_{11} \mathrm{H}_{12} \mathrm{O}_{2}$ & 176 & $66-68$ & Yellow & 89 \\
\hline 2 & $4-\mathrm{NO}_{2}$ & $\mathrm{C}_{6} \mathrm{H}_{5}$ & $\mathrm{C}_{15} \mathrm{H}_{11} \mathrm{NO}_{3}$ & 253 & $151-153$ & Light Brown & 81 \\
\hline 3 & $3-\mathrm{NO}_{2}$ & $\mathrm{C}_{6} \mathrm{H}_{5}$ & $\mathrm{C}_{15} \mathrm{H}_{11} \mathrm{NO}_{3}$ & 253 & $129-131$ & Yellow & 64 \\
\hline 4 & $\left.4-\mathrm{N}^{\circ} \mathrm{CH}_{3}\right)_{2}$ & $\mathrm{C}_{6} \mathrm{H}_{5}$ & $\mathrm{C}_{17} \mathrm{H}_{17} \mathrm{NO}$ & 251 & $63-65$ & Orange & 65 \\
\hline 5 & $\mathrm{H}$ & $3-\mathrm{NO}_{2} \mathrm{C}_{6} \mathrm{H}_{4}$ & $\mathrm{C}_{15} \mathrm{H}_{11} \mathrm{NO}_{3}$ & 253 & $118-120$ & Brown & 71 \\
\hline 6 & $2-\mathrm{NO}_{2}$ & $\mathrm{C}_{6} \mathrm{H}_{5}$ & $\mathrm{C}_{15} \mathrm{H}_{11} \mathrm{NO}_{3}$ & 253 & $131-134$ & Yellow & 75 \\
\hline 7 & $\mathrm{H}$ & $\mathrm{C}_{6} \mathrm{H}_{5}$ & $\mathrm{C}_{15} \mathrm{H}_{12} \mathrm{O}$ & 208 & $123-126$ & Yellow & 88 \\
\hline 8 & $4-\mathrm{NO}_{2}$ & $3-\mathrm{NO}_{2} \mathrm{C}_{6} \mathrm{H}_{4}$ & $\mathrm{C}_{15} \mathrm{H}_{10} \mathrm{~N}_{2} \mathrm{O}_{5}$ & 294 & $177-179$ & Brown & 81 \\
\hline 9 & $4-\mathrm{NO}_{2}$ & $2-\mathrm{NO}_{2} \mathrm{C}_{6} \mathrm{H}_{4}$ & $\mathrm{C}_{15} \mathrm{H}_{10} \mathrm{~N}_{2} \mathrm{O}_{5}$ & 294 & $181-184$ & Dark Brown & 73 \\
\hline 10 & $\mathrm{H}$ & $2-\mathrm{NO}_{2} \mathrm{C}_{6} \mathrm{H}_{4}$ & $\mathrm{C}_{15} \mathrm{H}_{11} \mathrm{NO}_{3}$ & 253 & $84-86$ & Brown & 63 \\
\hline
\end{tabular}


Table (2)The Physical Properties of Compound (11-20)

\begin{tabular}{|c|c|c|c|c|c|c|c|}
\hline $\begin{array}{c}\text { Comp. } \\
\text { No. }\end{array}$ & $\mathbf{R}$ & $\mathbf{R}_{\mathbf{1}}$ & $\begin{array}{c}\text { Molecular } \\
\text { formula }\end{array}$ & $\mathbf{M . ~ W t}$ & m.p. ${ }^{\circ} \mathbf{c}$ & Colour & $\begin{array}{c}\text { Yield } \\
\text { \% }\end{array}$ \\
\hline 11 & $4-\mathrm{OCH}_{3}$ & $\mathrm{CH}_{3}$ & $\mathrm{C}_{12} \mathrm{H}_{12} \mathrm{~N}_{2} \mathrm{O}_{2}$ & 216 & $253-255$ & Creamy & 72 \\
\hline 12 & $4-\mathrm{NO}_{2}$ & $\mathrm{C}_{6} \mathrm{H}_{5}$ & $\mathrm{C}_{16} \mathrm{H}_{11} \mathrm{~N}_{3} \mathrm{O}_{3}$ & 293 & $336-339$ & Dark Brown & 63 \\
\hline 13 & $3-\mathrm{NO}_{2}$ & $\mathrm{C}_{6} \mathrm{H}_{5}$ & $\mathrm{C}_{16} \mathrm{H}_{11} \mathrm{~N}_{3} \mathrm{O}_{3}$ & 293 & $297-299$ & Pale Brown & 77 \\
\hline 14 & $4-\mathrm{N}\left(\mathrm{CH}_{3}\right)_{2}$ & $\mathrm{C}_{6} \mathrm{H}_{5}$ & $\mathrm{C}_{18} \mathrm{H}_{17} \mathrm{~N}_{3} \mathrm{O}$ & 291 & $151-154$ & Pale Brown & 60 \\
\hline 15 & $\mathrm{H}$ & $3-\mathrm{NO}_{2} \mathrm{C}_{6} \mathrm{H}_{4}$ & $\mathrm{C}_{16} \mathrm{H}_{11} \mathrm{~N}_{3} \mathrm{O}_{3}$ & 293 & $263-265$ & Dark Brown & 86 \\
\hline 16 & $2-\mathrm{NO}_{2}$ & $\mathrm{C}_{6} \mathrm{H}_{5}$ & $\mathrm{C}_{16} \mathrm{H}_{11} \mathrm{~N}_{3} \mathrm{O}_{3}$ & 293 & $285-288$ & Brown & 81 \\
\hline 17 & $\mathrm{H}$ & $\mathrm{C}_{6} \mathrm{H}_{5}$ & $\mathrm{C}_{16} \mathrm{H}_{12} \mathrm{~N}_{2} \mathrm{O}$ & 248 & $222-225$ & Yellow & 85 \\
\hline 18 & $4-\mathrm{NO}_{2}$ & $3-\mathrm{NO}_{2} \mathrm{C}_{6} \mathrm{H}_{4}$ & $\mathrm{C}_{16} \mathrm{H}_{10} \mathrm{~N}_{4} \mathrm{O}_{5}$ & 338 & $285-289$ & Dark Brown & 76 \\
\hline 19 & $4-\mathrm{NO}_{2}$ & $2-\mathrm{NO}_{2} \mathrm{C}_{6} \mathrm{H}_{4}$ & $\mathrm{C}_{16} \mathrm{H}_{10} \mathrm{~N}_{4} \mathrm{O}_{5}$ & 338 & $315-318$ & Dark Brown & 70 \\
\hline 20 & $\mathrm{H}$ & $2-\mathrm{NO}_{2} \mathrm{C}_{6} \mathrm{H}_{4}$ & $\mathrm{C}_{16} \mathrm{H}_{11} \mathrm{~N}_{3} \mathrm{O}_{3}$ & 293 & $253-255$ & Brown & 80 \\
\hline
\end{tabular}

Table (3)The Physical Properties of Compound (21-30)

\begin{tabular}{|c|c|c|c|c|c|c|c|}
\hline $\begin{array}{c}\text { Comp. } \\
\text { No. }\end{array}$ & $\mathbf{R}$ & $\mathbf{R}_{\mathbf{1}}$ & $\begin{array}{c}\text { Molecular } \\
\text { formula }\end{array}$ & $\mathbf{M . ~ W t}$ & $\mathbf{m . p} \mathbf{c}^{\circ}$ & Colour & $\begin{array}{c}\text { Yield } \\
\text { \% }\end{array}$ \\
\hline 21 & $4-\mathrm{OCH}_{3}$ & $\mathrm{CH}_{3}$ & $\mathrm{C}_{24} \mathrm{H}_{17} \mathrm{~N}_{5} \mathrm{O}_{5}$ & 329 & $101-104$ & Light green & 90 \\
\hline 22 & $4-\mathrm{NO}_{2}$ & $\mathrm{C}_{6} \mathrm{H}_{5}$ & $\mathrm{C}_{20} \mathrm{H}_{15} \mathrm{NO}_{2}$ & 410 & $68-70$ & Brown & 81 \\
\hline 23 & $3-\mathrm{NO}_{2}$ & $\mathrm{C}_{6} \mathrm{H}_{5}$ & $\mathrm{C}_{24} \mathrm{H}_{14} \mathrm{~N}_{4} \mathrm{O}_{3}$ & 410 & $68-71$ & Dark Brown & 76 \\
\hline 24 & $4-\mathrm{N}\left(\mathrm{CH}_{3}\right)_{2}$ & $\mathrm{C}_{6} \mathrm{H}_{5}$ & $\mathrm{C}_{24} \mathrm{H}_{18} \mathrm{~N}_{4} \mathrm{O}_{3}$ & 407 & $62-65$ & Brown & 73 \\
\hline 25 & $\mathrm{H}$ & $3-\mathrm{NO}_{2} \mathrm{C}_{6} \mathrm{H}_{4}$ & $\mathrm{C}_{26} \mathrm{H}_{23} \mathrm{~N}_{4} \mathrm{O}$ & 410 & $82-85$ & Dark Brown & 63 \\
\hline 26 & $2-\mathrm{NO}_{2}$ & $\mathrm{C}_{6} \mathrm{H}_{5}$ & $\mathrm{C}_{24} \mathrm{H}_{18} \mathrm{~N}_{4} \mathrm{O}_{3}$ & 410 & $277-280$ & Brown & 77 \\
\hline 27 & $\mathrm{H}$ & $\mathrm{C}_{6} \mathrm{H}_{5}$ & $\mathrm{C}_{24} \mathrm{H}_{18} \mathrm{~N}_{4} \mathrm{O}$ & 365 & $162-165$ & Yellow & 88 \\
\hline 28 & $4-\mathrm{NO}_{2}$ & $3-\mathrm{NO}_{2} \mathrm{C}_{6} \mathrm{H}_{4}$ & $\mathrm{C}_{24} \mathrm{H}_{18} \mathrm{~N}_{4} \mathrm{O}_{3}$ & 455 & $88-90$ & Brown & 78 \\
\hline 29 & $4-\mathrm{NO}_{2}$ & $2-\mathrm{NO}_{2} \mathrm{C}_{6} \mathrm{H}_{4}$ & $\mathrm{C}_{24} \mathrm{H}_{18} \mathrm{~N}_{4} \mathrm{O}_{3}$ & 455 & $95-96$ & Light orange & 59 \\
\hline 30 & $\mathrm{H}$ & $2-\mathrm{NO}_{2} \mathrm{C}_{6} \mathrm{H}_{4}$ & $\mathrm{C}_{24} \mathrm{H}_{19} \mathrm{~N}_{3} \mathrm{O}$ & 410 & $75-78$ & Dark Yellow & 91 \\
\hline
\end{tabular}


Table (4) The Physical Properties of Compound (31-40)

\begin{tabular}{|c|c|c|c|c|c|c|c|}
\hline $\begin{array}{c}\text { Comp. } \\
\text { No. }\end{array}$ & $\mathbf{R}$ & $\mathbf{R}_{\mathbf{1}}$ & $\begin{array}{c}\text { Molecular } \\
\text { formula }\end{array}$ & $\mathbf{M . ~ W t}$ & m.p & Colour & $\begin{array}{c}\text { Yield } \\
\text { \% }\end{array}$ \\
\hline 31 & $4-\mathrm{OCH}_{3}$ & $\mathrm{CH}_{3}$ & $\mathrm{C}_{18} \mathrm{H}_{12} \mathrm{~N}_{5} \mathrm{O}_{5}$ & 378 & $118-120$ & Light Yellow & 85 \\
\hline 32 & $4-\mathrm{NO}_{2}$ & $\mathrm{C}_{6} \mathrm{H}_{5}$ & $\mathrm{C}_{22} \mathrm{H}_{14} \mathrm{~N}_{6} \mathrm{O}_{6}$ & 458 & $272-275$ & Light Brown & 67 \\
\hline 33 & $3-\mathrm{NO}_{2}$ & $\mathrm{C}_{6} \mathrm{H}_{5}$ & $\mathrm{C}_{22} \mathrm{H}_{14} \mathrm{~N}_{6} \mathrm{O}_{6}$ & 458 & $171-174$ & Pale Brown & 65 \\
\hline 34 & $4-\mathrm{N}\left(\mathrm{CH}_{3}\right)_{2}$ & $\mathrm{C}_{6} \mathrm{H}_{5}$ & $\mathrm{C}_{22} \mathrm{H}_{12} \mathrm{~N}_{6} \mathrm{O}_{6}$ & 456 & $148-151$ & Light green & 89 \\
\hline 35 & $\mathrm{H}$ & $3-\mathrm{NO}_{2} \mathrm{C}_{6} \mathrm{H}_{4}$ & $\mathrm{C}_{22} \mathrm{H}_{14} \mathrm{~N}_{6} \mathrm{O}_{6}$ & 458 & $168-170$ & Light green & 63 \\
\hline 36 & $2-\mathrm{NO}_{2}$ & $\mathrm{C}_{6} \mathrm{H}_{5}$ & $\mathrm{C}_{22} \mathrm{H}_{14} \mathrm{~N}_{6} \mathrm{O}_{6}$ & 458 & $167-170$ & Dark Yellow & 72 \\
\hline 37 & $\mathrm{H}$ & $\mathrm{C}_{6} \mathrm{H}_{5}$ & $\mathrm{C}_{22} \mathrm{H}_{15} \mathrm{~N}_{5} \mathrm{O}_{4}$ & 413 & $169-172$ & Yellow & 82 \\
\hline 38 & $4-\mathrm{NO}_{2}$ & $3-\mathrm{NO}_{2} \mathrm{C}_{6} \mathrm{H}_{4}$ & $\mathrm{C}_{22} \mathrm{H}_{13} \mathrm{~N}_{7} \mathrm{O}_{8}$ & 503 & $206-209$ & Dark Brown & 75 \\
\hline 39 & $4-\mathrm{NO}_{2}$ & $2-\mathrm{NO}_{2} \mathrm{C}_{6} \mathrm{H}_{4}$ & $\mathrm{C}_{22} \mathrm{H}_{13} \mathrm{~N}_{7} \mathrm{O}_{8}$ & 503 & $217-220$ & Dark Brown & 81 \\
\hline 40 & $\mathrm{H}$ & $2-\mathrm{NO}_{2} \mathrm{C}_{6} \mathrm{H}_{4}$ & $\mathrm{C}_{22} \mathrm{H}_{14} \mathrm{~N}_{6} \mathrm{O}_{6}$ & 458 & $159-161$ & Brown & 66 \\
\hline
\end{tabular}

Table (5) The Physical Properties of Compound (41-50)

\begin{tabular}{|c|c|c|c|c|c|c|c|}
\hline $\begin{array}{c}\text { Comp. } \\
\text { No. }\end{array}$ & $\mathbf{R}$ & $\mathbf{R}_{\mathbf{1}}$ & $\begin{array}{c}\text { Molecular } \\
\text { formula }\end{array}$ & $\mathbf{M}$. Wt & mp c & Colour & $\begin{array}{c}\text { Yield } \\
\text { \% }\end{array}$ \\
\hline 41 & $4-\mathrm{OCH}_{3}$ & $\mathrm{CH}_{3}$ & $\mathrm{C}_{22} \mathrm{H}_{17} \mathrm{~N}_{3} \mathrm{O}_{3} \mathrm{~S}$ & 403 & $180-183$ & Creamy & 79 \\
\hline 42 & $4-\mathrm{NO}_{2}$ & $\mathrm{C}_{6} \mathrm{H}_{5}$ & $\mathrm{C}_{26} \mathrm{H}_{20} \mathrm{~N}_{4} \mathrm{O}_{4} \mathrm{~S}$ & 484 & $284-286$ & $\begin{array}{c}\text { Light } \\
\text { Brown }\end{array}$ & 81 \\
\hline 43 & $3-\mathrm{NO}_{2}$ & $\mathrm{C}_{6} \mathrm{H}_{5}$ & $\mathrm{C}_{26} \mathrm{H}_{20} \mathrm{~N}_{4} \mathrm{O}_{4} \mathrm{~S}$ & 484 & $275-278$ & Brown & 78 \\
\hline 44 & $\left.4-\mathrm{N}_{2} \mathrm{CH}_{3}\right)_{2}$ & $\mathrm{C}_{6} \mathrm{H}_{5}$ & $\mathrm{C}_{28} \mathrm{H}_{25} \mathrm{~N}_{4} \mathrm{O}_{2} \mathrm{~S}$ & 481 & $273-275$ & Yellow & 85 \\
\hline 45 & $\mathrm{H}$ & $3-\mathrm{NO}_{2} \mathrm{C}_{6} \mathrm{H}_{4}$ & $\mathrm{C}_{26} \mathrm{H}_{20} \mathrm{~N}_{4} \mathrm{O}_{4} \mathrm{~S}$ & 484 & $103-105$ & $\begin{array}{c}\text { Light } \\
\text { green }\end{array}$ & 81 \\
\hline 46 & $2-\mathrm{NO}_{2}$ & $\mathrm{C}_{6} \mathrm{H}_{5}$ & $\mathrm{C}_{26} \mathrm{H}_{20} \mathrm{~N}_{4} \mathrm{O}_{4} \mathrm{~S}$ & 484 & $245-248$ & $\begin{array}{c}\text { Dark } \\
\text { Brown }\end{array}$ & 80 \\
\hline 47 & $\mathrm{H}$ & $\mathrm{C}_{6} \mathrm{H}_{5}$ & $\mathrm{C}_{26} \mathrm{H}_{21} \mathrm{~N}_{3} \mathrm{O}_{2} \mathrm{~S}$ & 439 & $76-78$ & Yellow & 88 \\
\hline 48 & $4-\mathrm{NO}_{2}$ & $3-\mathrm{NO}_{2} \mathrm{C}_{6} \mathrm{H}_{4}$ & $\mathrm{C}_{26} \mathrm{H}_{20} \mathrm{~N}_{4} \mathrm{O}_{4} \mathrm{~S}$ & 484 & $88-90$ & Brown & 66 \\
\hline 49 & $4-\mathrm{NO}_{2}$ & $2-\mathrm{NO}_{2} \mathrm{C}_{6} \mathrm{H}_{4}$ & $\mathrm{C}_{26} \mathrm{H}_{20} \mathrm{~N}_{4} \mathrm{O}_{4} \mathrm{~S}$ & 529 & $76-78$ & Yellow & 91 \\
\hline 50 & $\mathrm{H}$ & $2-\mathrm{NO}_{2} \mathrm{C}_{6} \mathrm{H}_{4}$ & $\mathrm{C}_{26} \mathrm{H}_{20} \mathrm{~N}_{4} \mathrm{O}_{4} \mathrm{~S}$ & 484 & $95-98$ & $\begin{array}{c}\text { Dark } \\
\text { Brown }\end{array}$ & 73 \\
\hline
\end{tabular}


Table (6) The Physical Properties of Compound (51-60)

\begin{tabular}{|c|c|c|c|c|c|c|c|}
\hline $\begin{array}{c}\text { Comp. } \\
\text { No. }\end{array}$ & $\mathbf{R}$ & $\mathbf{R}_{\mathbf{1}}$ & $\begin{array}{c}\text { Molecular } \\
\text { formula }\end{array}$ & $\mathbf{M . ~ W t}$ & $\mathbf{m p}^{\circ} \mathbf{c}$ & Colour & Yield \% \\
\hline 51 & $4-\mathrm{OCH}_{3}$ & $\mathrm{CH}_{3}$ & $\mathrm{C}_{20} \mathrm{H}_{17} \mathrm{~N}_{5} \mathrm{O}_{8}$ & 483 & $95-98$ & Light Yellow & 65 \\
\hline 52 & $4-\mathrm{NO}_{2}$ & $\mathrm{C}_{6} \mathrm{H}_{5}$ & $\mathrm{C}_{24} \mathrm{H}_{16} \mathrm{~N}_{6} \mathrm{O}_{9}$ & 532 & $111-114$ & Brown & 55 \\
\hline 53 & $3-\mathrm{NO}_{2}$ & $\mathrm{C}_{6} \mathrm{H}_{5}$ & $\mathrm{C}_{24} \mathrm{H}_{16} \mathrm{~N}_{6} \mathrm{O}_{9}$ & 532 & $333-335$ & Pale Brown & 71 \\
\hline 54 & $4-\mathrm{N}\left(\mathrm{CH}_{3}\right)_{2}$ & $\mathrm{C}_{6} \mathrm{H}_{5}$ & $\mathrm{C}_{26} \mathrm{H}_{22} \mathrm{~N}_{6} \mathrm{O}_{7}$ & 530 & $85-87$ & Red & 83 \\
\hline 55 & $\mathrm{H}$ & $3-\mathrm{NO}_{2} \mathrm{C}_{6} \mathrm{H}_{4}$ & $\mathrm{C}_{24} \mathrm{H}_{16} \mathrm{~N}_{6} \mathrm{O}_{9}$ & 532 & $148-151$ & Light green & 67 \\
\hline 56 & $2-\mathrm{NO}_{2}$ & $\mathrm{C}_{6} \mathrm{H}_{5}$ & $\mathrm{C}_{24} \mathrm{H}_{16} \mathrm{~N}_{6} \mathrm{O}_{9}$ & 532 & $327-330$ & Dark Brown & 74 \\
\hline 57 & $\mathrm{H}$ & $\mathrm{C}_{6} \mathrm{H}_{5}$ & $\mathrm{C}_{24} \mathrm{H}_{17} \mathrm{~N}_{5} \mathrm{O}_{7}$ & 487 & $226-229$ & Yellow & 81 \\
\hline 58 & $4-\mathrm{NO}_{2}$ & $3-\mathrm{NO}_{2} \mathrm{C}_{6} \mathrm{H}_{4}$ & $\mathrm{C}_{24} \mathrm{H}_{15} \mathrm{~N}_{5} \mathrm{O}_{11}$ & 577 & $267-269$ & Brown & 85 \\
\hline 59 & $4-\mathrm{NO}_{2}$ & $2-\mathrm{NO}_{2} \mathrm{C}_{6} \mathrm{H}_{4}$ & $\mathrm{C}_{24} \mathrm{H}_{15} \mathrm{~N}_{5} \mathrm{O}_{11}$ & 577 & $287-290$ & Dark Brown & 87 \\
\hline 60 & $\mathrm{H}$ & $2-\mathrm{NO}_{2} \mathrm{C}_{6} \mathrm{H}_{4}$ & $\mathrm{C}_{24} \mathrm{H}_{16} \mathrm{~N}_{6} \mathrm{O}_{9}$ & 532 & $181-184$ & Light Brown & 77 \\
\hline
\end{tabular}

\section{RESULTS AND DISCUSSIONS}

New schiff's bases were synthesized by reactions of pyrimidines compounds with different aromatic amine in abs. EtOH with some drops of glacial acetic acid. The FTIR spectra of Schiff's bases compounds (21-40) showed the absence of peak of carbonyl but a new peak was appeared at $(1588-1656) \mathrm{cm}^{-1}$ which is attributed to a new $(\mathrm{C}=\mathrm{N})$ group as in [19]. Table $(7,8)$ showed some spectral data. Figure (1), (3) showed IR spectrum for compounds (29, 35).

Thiazolidine compounds were prepared from the reaction of Schiff's bases (21-30) with thioglycolic acid in abs. EtOH. FTIR spectrum showed new peak appeared at (669-776) which is attributed to a new (C-S) group and another peak appeared at $(1555-1643) \mathrm{cm}^{-1}$ is attributed to $(\mathrm{C}=\mathrm{N})$ group as in [20], Table (9) referred to some spectral data. Figure (5), showed IR spectrum for compound (49). 1,3- Oxazepine compound prepared from the reaction of Schiff's bases (31-40) with maleic anhydride in abs. EtOH. FT-IR spectrum showed a new peak at (1684-1711) $\mathrm{cm}^{-1}$ that is attributed to (O-C) Lactane and another peak appeared at $\left(1620 \_1654\right) \mathrm{cm}^{-1}$ attributed to $(\mathrm{N}-\mathrm{C}=\mathrm{O})$ Lactame. Table (9) showed some spectral data [18]. Figure (7) showed IR spectrum for compound (54).

The ${ }^{1} \mathrm{H}-\mathrm{NMR}$ spectrum showed these bands:

Compound (31); ${ }^{1} \mathrm{H}-\mathrm{NMR}$ (DMSO, 60MHz): $\delta=7.18-8.77(\mathrm{~m}, 7 \mathrm{H}, \mathrm{Ar}-\mathrm{H}), 7.02(\mathrm{~s}, \mathrm{C}=\mathrm{C}-\mathrm{H}, 1 \mathrm{H}), 5.39$ (b, N-H,1H),1.59(s, O- $\left.\mathrm{CH}_{3}\right), 1.1\left(\mathrm{~s},-\mathrm{CH}_{3}, 3 \mathrm{H}\right)$. [19].

Figure (2) showed NMR spectrum of compound (31).

Compound (29): ${ }^{1} \mathrm{H}-\mathrm{NMR}$ (DMSO,60MHz): $\delta=6.43-7.68(\mathrm{dd}, \mathrm{Ar}-\mathrm{H}), 5.94(\mathrm{~m}, 5 \mathrm{H}, \mathrm{Ar}-\mathrm{H}), 2.4(\mathrm{~b}, 1 \mathrm{H}$, $\mathrm{N}-\mathrm{H})$.

Figure (2) showed NMR spectrum of compound (29).

Compound (49): ${ }^{1} \mathrm{H}-\mathrm{NMR}$ (DMSO,60MHz): $\delta=6.43-7.68$ (dd,8H,Ar-H), 5.95 (m,5H,Ar-H,C=C-H), $2.83\left(\mathrm{~b},{ }^{1} \mathrm{H}, \mathrm{N}-\mathrm{H}\right), 2.4-2.67\left(\mathrm{~b}, 5 \mathrm{H}, \mathrm{CH}_{3}, \mathrm{CH}_{2}\right),[20]$.

Figure (6) showed NMR spectrum of compound (49).

Compound (54): ${ }^{1} \mathrm{HNMR}$ (DMSO,60MHz): $\delta=6.99-8.77(\mathrm{~m}, 12 \mathrm{H}, \mathrm{Ar}-\mathrm{H}), 6.14-6.30(\mathrm{~d}, 3 \mathrm{H}, \mathrm{C}=\mathrm{C}-\mathrm{H})$, $3.01(\mathrm{~s}, 1 \mathrm{H}, \mathrm{N}-\mathrm{H}), 1.16\left(\mathrm{~s}, 3 \mathrm{H}, \mathrm{CH}_{3}\right)$,

Figure (8) showed NMR spectrum of compound (54). 
Table (7) Some Spectral Data of Compounds (21-30)

\begin{tabular}{|c|c|c|c|c|c|c|c|c|}
\hline $\begin{array}{c}\text { Comp. } \\
\text { No }\end{array}$ & $\mathbf{R}$ & $\mathbf{R}_{1}$ & N-H & C-H Ar & C-H Alph. & $\mathrm{C}=\mathbf{O}$ & $\mathbf{C}=\mathbf{N}$ & Others \\
\hline 21 & $4-\mathrm{OCH}_{3}$ & $\mathrm{CH}_{3}$ & 3387 & 3097 & 2851 & 1700 & 1638 & $\begin{array}{c}1551 \mathrm{C}=\mathrm{C} \\
1170 \mathrm{C}-\mathrm{O}-\mathrm{C} \text { Asy } \\
1021 \mathrm{C}-\mathrm{O}-\mathrm{C} \text { Sym }\end{array}$ \\
\hline 22 & $4-\mathrm{NO}_{2}$ & $\mathrm{C}_{6} \mathrm{H}_{5}$ & 3320 & 3115 & 2905 & 1684 & 1642 & $\begin{array}{c}1542 \mathrm{NO}_{2} \mathrm{Asy} \\
1351 \mathrm{NO}_{2} \mathrm{Sym} \\
1580 \mathrm{C}=\mathrm{C}\end{array}$ \\
\hline 23 & $3-\mathrm{NO}_{2}$ & $\mathrm{C}_{6} \mathrm{H}_{5}$ & 3352 & 3052 & 2928 & 1710 & 1655 & $\begin{array}{c}1485 \mathrm{NO}_{2} \mathrm{Asy} \\
1351 \mathrm{NO}_{2} \mathrm{Asy} \\
1588 \mathrm{C}=\mathrm{C}\end{array}$ \\
\hline 24 & $4-\mathrm{N}\left(\mathrm{CH}_{3}\right)_{2}$ & $\mathrm{C}_{6} \mathrm{H}_{5}$ & 3216 & 3033 & 2917 & 1675 & 1588 & $1520 \mathrm{C}=\mathrm{C}$ \\
\hline 25 & $\mathrm{H}$ & $3-\mathrm{NO}_{2} \mathrm{C}_{6} \mathrm{H}_{4}$ & 3218 & 3081 & 2908 & 1685 & 1639 & $\begin{array}{c}1553 \mathrm{NO}_{2} \mathrm{Asy} \\
1356 \mathrm{NO}_{2} \mathrm{Sym} \\
1578 \mathrm{C}=\mathrm{C}\end{array}$ \\
\hline 26 & $2-\mathrm{NO}_{2}$ & $\mathrm{C}_{6} \mathrm{H}_{5}$ & 3219 & 3058 & 2968 & 1730 & 1655 & $\begin{array}{c}1588 \mathrm{C}=\mathrm{C} \\
1526 \mathrm{NO}_{2} \mathrm{Asy} \\
1345 \mathrm{NO}_{2} \text { Sym }\end{array}$ \\
\hline 27 & $\mathrm{H}$ & $\mathrm{C}_{6} \mathrm{H}_{5}$ & 3353 & 3105 & 2999 & 1725 & 1656 & $1599 \mathrm{C}=\mathrm{C}$ \\
\hline 28 & $4-\mathrm{NO}_{2}$ & $3-\mathrm{NO}_{2} \mathrm{C}_{6} \mathrm{H}_{4}$ & 3343 & 3110 & 2942 & 1686 & 1637 & $\begin{array}{c}1578 \mathrm{C}=\mathrm{C} \\
1524 \mathrm{NO}_{2} \mathrm{Asy} \\
1404 \mathrm{NO}_{2} \mathrm{Sym}\end{array}$ \\
\hline 29 & $4-\mathrm{NO}_{2}$ & $2-\mathrm{NO}_{2} \mathrm{C}_{6} \mathrm{H}_{4}$ & 3214 & 3018 & 2854 & 1718 & 1640 & $\begin{array}{c}1551 \mathrm{C}=\mathrm{C} \\
1510 \mathrm{NO}_{2} \mathrm{Asy} \\
1354 \mathrm{NO}_{2} \mathrm{Sym}\end{array}$ \\
\hline 30 & $\mathrm{H}$ & $2-\mathrm{NO}_{2} \mathrm{C}_{6} \mathrm{H}_{4}$ & 3363 & 3031 & 2922 & 1686 & 1642 & $\begin{array}{c}1588 \mathrm{C}=\mathrm{C} \\
1524 \mathrm{NO}_{2} \mathrm{Asy} \\
1406 \mathrm{NO}_{2} \mathrm{Sym}\end{array}$ \\
\hline
\end{tabular}

Table (8) Some Spectral Data of Compounds (31-40)

\begin{tabular}{|c|c|c|c|c|c|c|c|}
\hline Comp. No. & $\mathbf{R}$ & $\mathbf{R}_{\mathbf{1}}$ & $\mathbf{N}-\mathbf{H}$ & $\mathbf{C}-\mathbf{H} \mathbf{A r}$ & $\mathbf{C}-\mathbf{H}$ alph. & $\mathbf{C = N}$ & Others \\
\hline 31 & $4-\mathrm{OCH}_{3}$ & $\mathrm{CH}_{3}$ & 3329 & 3158 & 2985 & 1630 & $\begin{array}{c}1403 \mathrm{COC} \text { Asy } \\
1550 \mathrm{NO}_{2} \mathrm{Asy} \\
1333 \mathrm{NO}_{2} \mathrm{Sym}\end{array}$ \\
\hline 32 & $4-\mathrm{NO}_{2}$ & $\mathrm{C}_{6} \mathrm{H}_{5}$ & 3353 & 3090 & 2850 & 1640 & $\begin{array}{c}1526 \mathrm{NO}_{2} \mathrm{Asy} \\
1326 \mathrm{NO}_{2} \mathrm{Sym}\end{array}$ \\
\hline 33 & $3-\mathrm{NO}_{2}$ & $\mathrm{C}_{6} \mathrm{H}_{5}$ & 3325 & 3099 & 2845 & 1620 & $\begin{array}{c}1504 \mathrm{NO}_{2} \mathrm{Asy} \\
1326 \mathrm{NO}_{2} \mathrm{Sym}\end{array}$ \\
\hline 34 & $\left.4-\mathrm{N}_{(\mathrm{CH}}\right)_{2}$ & $\mathrm{C}_{6} \mathrm{H}_{5}$ & 3326 & 3096 & 2902 & 1592 & $\begin{array}{c}1504 \mathrm{NO}_{2} \mathrm{Asy} \\
1379 \mathrm{NO}_{2} \mathrm{Sym}\end{array}$ \\
\hline 35 & $\mathrm{H}$ & $3-\mathrm{NO}_{2} \mathrm{C}_{6} \mathrm{H}_{4}$ & 3324 & 3099 & 2935 & 1618 & $\begin{array}{l}1490 \mathrm{NO}_{2} \mathrm{Asy} \\
1325 \mathrm{NO}_{2} \mathrm{Sym}\end{array}$ \\
\hline 36 & $2-\mathrm{NO}_{2}$ & $\mathrm{C}_{6} \mathrm{H}_{5}$ & 3326 & 3125 & 2850 & 1605 & $\begin{array}{l}1556 \mathrm{NO}_{2} \mathrm{Asy} \\
1328 \mathrm{NO}_{2} \mathrm{Sym}\end{array}$ \\
\hline 37 & $\mathrm{H}$ & $\mathrm{C}_{6} \mathrm{H}_{5}$ & 3175 & 3072 & 2833 & 1623 & $\begin{array}{l}1507 \mathrm{NO}_{2} \mathrm{Asy} \\
1330 \mathrm{NO}_{2} \mathrm{Sym}\end{array}$ \\
\hline 39 & $4-\mathrm{NO}_{2}$ & $3-\mathrm{NO}_{2} \mathrm{C}_{6} \mathrm{H}_{4}$ & 3331 & 3166 & 2878 & 1627 & $\begin{array}{l}1555 \mathrm{NO}_{2} \mathrm{Asy} \\
1328 \mathrm{NO}_{2} \mathrm{Sym}\end{array}$ \\
\hline 40 & $4-\mathrm{NO}_{2}$ & $2-\mathrm{NO}_{2} \mathrm{C}_{6} \mathrm{H}_{4}$ & 3326 & 3024 & 2940 & 1633 & $\begin{array}{l}1552 \mathrm{NO}_{2} \mathrm{Asy} \\
1326 \mathrm{NO}_{2} \mathrm{Sym}\end{array}$ \\
\hline $\mathrm{H}$ & $2-\mathrm{NO}_{2} \mathrm{C}_{6} \mathrm{H}_{4}$ & 3326 & 3043 & 2953 & 1621 & $\begin{array}{l}1504 \mathrm{NO}_{2} \mathrm{Asy} \\
1324 \mathrm{NO}_{2} \mathrm{Sym}\end{array}$ \\
\hline
\end{tabular}


Table (9) Some Spectral Data of Compounds (41- 50)

\begin{tabular}{|c|c|c|c|c|c|c|c|c|c|c|}
\hline $\begin{array}{c}\text { Comp. } \\
\text { No }\end{array}$ & $\mathbf{R}$ & $\mathbf{R}_{\mathbf{1}}$ & N-H & $\begin{array}{c}\text { C-H } \\
\text { Ar }\end{array}$ & $\begin{array}{l}\text { C-H } \\
\text { Alph. }\end{array}$ & $\mathrm{C}=\mathrm{O}$ & $\mathbf{C}=\mathbf{N}$ & $\mathrm{C}=\mathrm{C}$ & $\mathrm{C}=\mathrm{S}$ & Others \\
\hline 41 & $4-\mathrm{OCH}_{3}$ & $\mathrm{CH}_{3}$ & 3200 & 3030 & 2850 & 1684 & 1578 & 1460 & 673 & $\begin{array}{l}1109 \text { cocAsy } \\
1027 \text { coc Sym }\end{array}$ \\
\hline 42 & $4-\mathrm{NO}_{2}$ & $\mathrm{C}_{6} \mathrm{H}_{5}$ & 3268 & 3015 & 2919 & 1640 & 1574 & 1490 & 676 & $\begin{array}{l}1498 \mathrm{NO}_{2} \text { Asy } \\
1340 \mathrm{NO}_{2} \text { Sym }\end{array}$ \\
\hline 43 & $3-\mathrm{NO}_{2}$ & $\mathrm{C}_{6} \mathrm{H}_{5}$ & 3383 & 3075 & 2980 & 1655 & 1595 & 1479 & 665 & $\begin{array}{l}1488 \mathrm{NO}_{2} \text { Asy } \\
1354 \mathrm{NO}_{2} \text { Sym }\end{array}$ \\
\hline 44 & $4-\mathrm{N}\left(\mathrm{CH}_{3}\right)_{2}$ & $\mathrm{C}_{6} \mathrm{H}_{5}$ & 3216 & 3091 & 2981 & 1684 & 1573 & 1488 & 673 & -------------- \\
\hline 45 & $\mathrm{H}$ & $3-\mathrm{NO}_{2} \mathrm{C}_{6} \mathrm{H}_{4}$ & 3215 & 3129 & 2910 & 1702 & 1555 & 1468 & 686 & $\begin{array}{l}1504 \mathrm{NO}_{2} \text { Asy } \\
1378 \mathrm{NO}_{2} \text { Sym }\end{array}$ \\
\hline 46 & $2-\mathrm{NO}_{2}$ & $\mathrm{C}_{6} \mathrm{H}_{5}$ & 3442 & 3109 & 2920 & 1685 & 1576 & 1458 & 776 & $\begin{array}{l}1374 \mathrm{NO}_{2} \mathrm{Sym} \\
1203 \mathrm{NO}_{2} \text { Sym } \\
\end{array}$ \\
\hline 47 & $\mathrm{H}$ & $\mathrm{C}_{6} \mathrm{H}_{5}$ & 3374 & 3051 & 2950 & 1700 & 1610 & 1578 & 669 & --------------- \\
\hline 48 & $4-\mathrm{NO}_{2}$ & $3-\mathrm{NO}_{2} \mathrm{C}_{6} \mathrm{H}_{4}$ & 3264 & 3119 & 2870 & 1675 & 1633 & 1569 & 672 & $\begin{array}{l}1389 \mathrm{NO}_{2} \text { Sym } \\
1212 \mathrm{NO}_{2} \text { Sym }\end{array}$ \\
\hline 49 & $4-\mathrm{NO}_{2}$ & $2-\mathrm{NO}_{2} \mathrm{C}_{6} \mathrm{H}_{4}$ & 3319 & 3080 & 2830 & 1695 & 1641 & 1578 & 669 & $\begin{array}{l}1425 \mathrm{NO}_{2} \mathrm{Sym} \\
1357 \mathrm{NO}_{2} \mathrm{Sym}\end{array}$ \\
\hline 50 & $\mathrm{H}$ & $2-\mathrm{NO}_{2} \mathrm{C}_{6} \mathrm{H}_{4}$ & 3374 & 3120 & 2920 & 1685 & 1643 & 1569 & 672 & $\begin{array}{l}1389 \mathrm{NO}_{2} \text { Sym } \\
1222 \mathrm{NO}_{2} \text { Sym } \\
\end{array}$ \\
\hline
\end{tabular}

Table (10) Some Spectral Data of Compounds (41-50)

\begin{tabular}{|c|c|c|c|c|c|c|c|c|c|}
\hline $\begin{array}{c}\text { Comp. } \\
\text { No. }\end{array}$ & $\mathbf{R}$ & $\mathbf{R}_{1}$ & N-H & C-H Ar & $\begin{array}{l}\text { C-H } \\
\text { Alph. }\end{array}$ & $\begin{array}{c}\mathrm{O} \\
-\mathrm{C}- \\
\text { Lacton }\end{array}$ & $\begin{array}{c}\mathrm{O} \\
-\mathrm{C}- \\
\text { Lactam }\end{array}$ & $\mathbf{C}=\mathbf{N}$ & Others \\
\hline 51 & $4-\mathrm{OCH}_{3}$ & $\mathrm{CH}_{3}$ & 3328 & 3055 & 2930 & 1703 & 1621 & 1579 & $\begin{array}{c}1501 \mathrm{NO}_{2} \mathrm{Asy} \\
1327 \mathrm{NO}_{2} \mathrm{Sym} \\
1418 \mathrm{C}=\mathrm{C} \mathrm{Ar} \\
1170 \mathrm{C}-\mathrm{O}-\mathrm{C} \text { Asy } \\
1064 \mathrm{C}-\mathrm{O}-\mathrm{C} \text { Sym }\end{array}$ \\
\hline 52 & $4-\mathrm{NO}_{2}$ & $\mathrm{C}_{6} \mathrm{H}_{5}$ & 3285 & 3045 & 2855 & 1695 & 1630 & 1559 & $\begin{array}{c}1504 \mathrm{NO}_{2} \mathrm{Asy} \\
1328 \mathrm{NO}_{2} \mathrm{Sym} \\
1416 \mathrm{C}=\mathrm{C} \mathrm{Ar}\end{array}$ \\
\hline 53 & $3-\mathrm{NO}_{2}$ & $\mathrm{C}_{6} \mathrm{H}_{5}$ & 3351 & 3061 & 2830 & 1695 & 1644 & 1605 & $\begin{array}{c}1509 \mathrm{NO}_{2} \mathrm{Asy} \\
1324 \mathrm{NO}_{2} \mathrm{Sym} \\
1416 \mathrm{C}=\mathrm{C} \mathrm{Ar}\end{array}$ \\
\hline 54 & $4-\mathrm{N}\left(\mathrm{CH}_{3}\right)_{2}$ & $\mathrm{C}_{6} \mathrm{H}_{5}$ & 3326 & 3021 & 2918 & 1711 & 1620 & 1576 & $\begin{array}{c}1506 \mathrm{NO}_{2} \mathrm{Asy} \\
1325 \mathrm{NO}_{2} \mathrm{Sym} \\
1427 \mathrm{C}=\mathrm{C} \mathrm{Ar}\end{array}$ \\
\hline 55 & $\mathrm{H}$ & $3-\mathrm{NO}_{2} \mathrm{C}_{6} \mathrm{H}_{4}$ & 3327 & 3103 & 2930 & 1712 & 1620 & 1578 & $\begin{array}{c}1501 \mathrm{NO}_{2} \mathrm{Asy} \\
1325 \mathrm{NO}_{2} \mathrm{Sym} \\
1422 \mathrm{C}=\mathrm{C} \mathrm{Ar}\end{array}$ \\
\hline 56 & $2-\mathrm{NO}_{2}$ & $\mathrm{C}_{6} \mathrm{H}_{5}$ & 3251 & 3056 & 2969 & 1708 & 1654 & 1587 & $\begin{array}{c}1525 \mathrm{NO}_{2} \mathrm{Asy} \\
1345 \mathrm{NO}_{2} \mathrm{Sym} \\
1457 \mathrm{C}=\mathrm{C} \mathrm{Ar}\end{array}$ \\
\hline 57 & $\mathrm{H}$ & $\mathrm{C}_{6} \mathrm{H}_{5}$ & 3316 & 3174 & 2908 & 1700 & 1653 & 1570 & $\begin{array}{l}1507 \mathrm{NO}_{2} \mathrm{Asy} \\
1301 \mathrm{NO}_{2} \text { Sym } \\
1457 \mathrm{C}=\mathrm{C} \text { AR }\end{array}$ \\
\hline 58 & $4-\mathrm{NO}_{2}$ & $3-\mathrm{NO}_{2} \mathrm{C}_{6} \mathrm{H}_{4}$ & 3354 & 3080 & 2968 & 1684 & 1635 & 1552 & $\begin{array}{c}1524 \mathrm{NO}_{2} \mathrm{Asy} \\
1388 \mathrm{NO}_{2} \mathrm{Sym} \\
1461 \mathrm{C}=\mathrm{C} \mathrm{Ar}\end{array}$ \\
\hline 59 & $4-\mathrm{NO}_{2}$ & $2-\mathrm{NO}_{2} \mathrm{C}_{6} \mathrm{H}_{4}$ & 3293 & 3085 & 2918 & 1690 & 1640 & 1553 & $\begin{array}{c}1510 \mathrm{NO}_{2} \mathrm{Asy} \\
1377 \mathrm{NO}_{2} \mathrm{Sym} \\
1459 \mathrm{C}=\mathrm{C} \mathrm{Ar}\end{array}$ \\
\hline 60 & $\mathrm{H}$ & $2-\mathrm{NO}_{2} \mathrm{C}_{6} \mathrm{H}_{4}$ & 3281 & 3078 & 2855 & 1688 & 1651 & 1563 & $\begin{array}{c}1501 \mathrm{NO}_{2} \mathrm{Asy} \\
1328 \mathrm{NO}_{2} \mathrm{Sym} \\
1458 \mathrm{C}=\mathrm{C} \mathrm{Ar}\end{array}$ \\
\hline
\end{tabular}




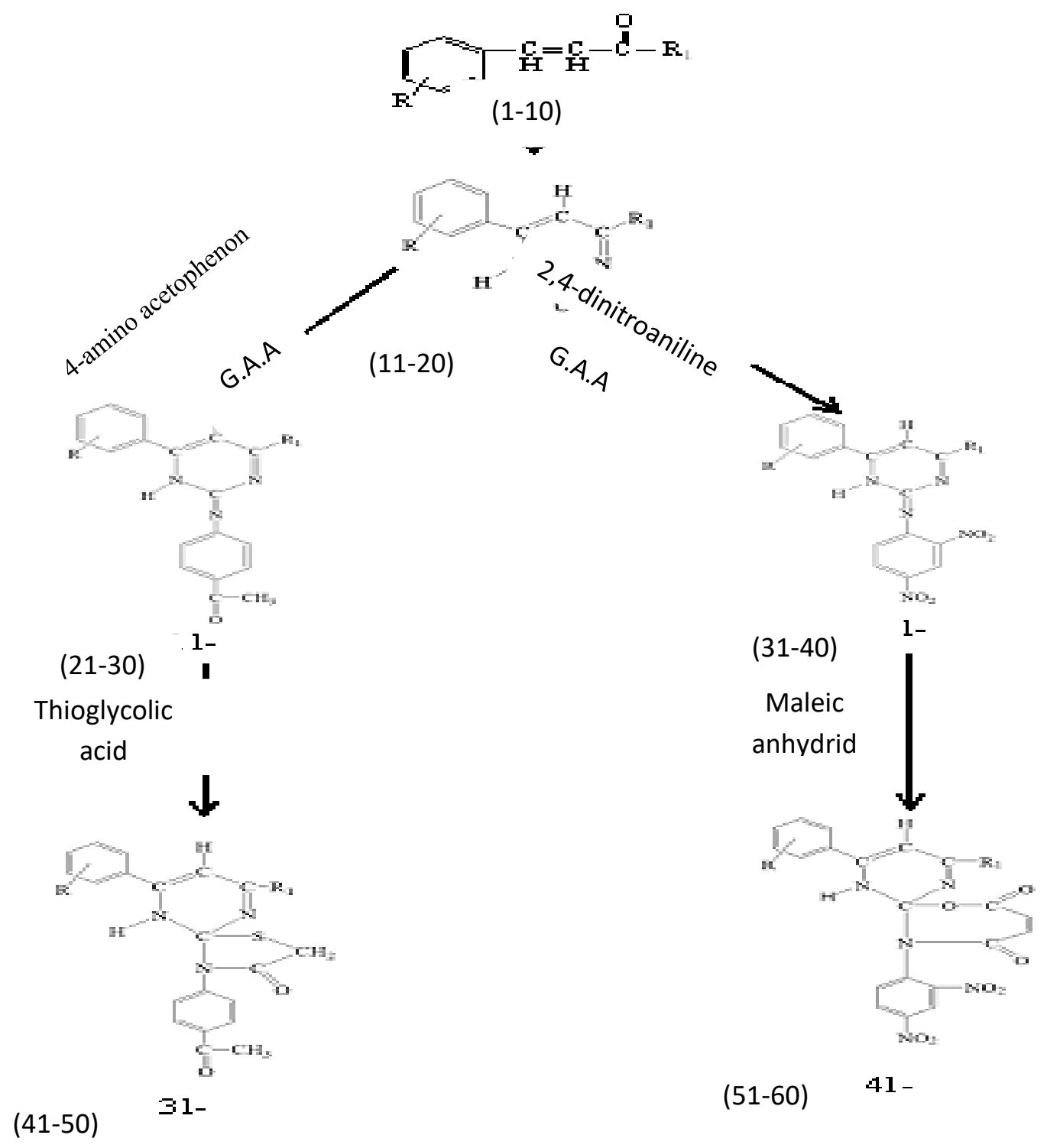

$\mathrm{R}=4-\mathrm{OCH}_{2}, 4-\mathrm{NO}_{2}, 4-\mathrm{N}\left(\mathrm{CH}_{3}\right)_{2}, 2-\mathrm{NO}_{2}, \mathrm{H}$

$\mathrm{R} 1=\mathrm{CH}_{3}, 3-\mathrm{NO}_{2} \mathrm{C}_{6} \mathrm{H}_{5}, 2-\mathrm{NO}_{2} \mathrm{C}_{6} \mathrm{H}_{5}$

Scheme (1) illustrates the prepared compounds (1-60) 


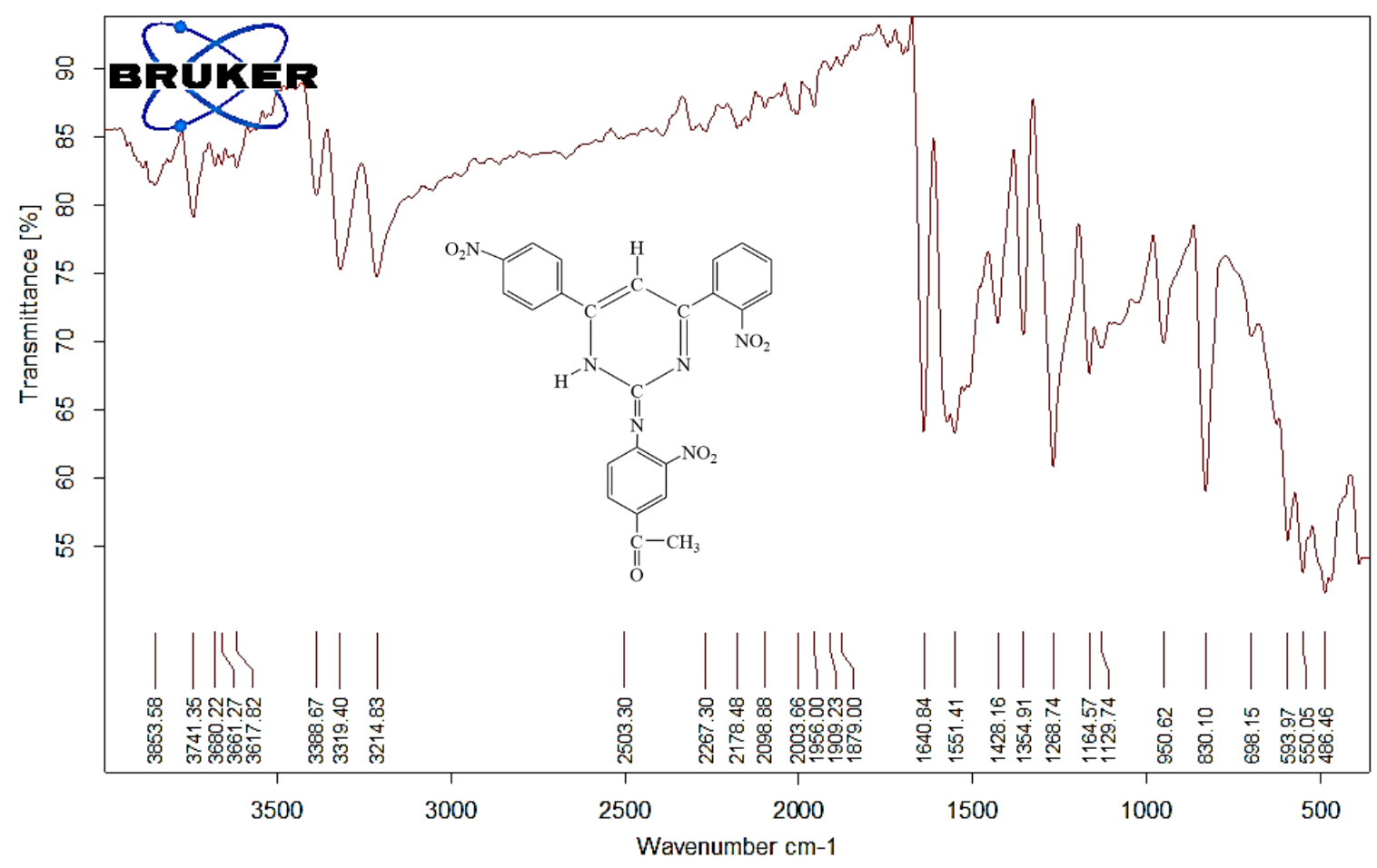

Fig (1): IR spectrum of the compound (29)

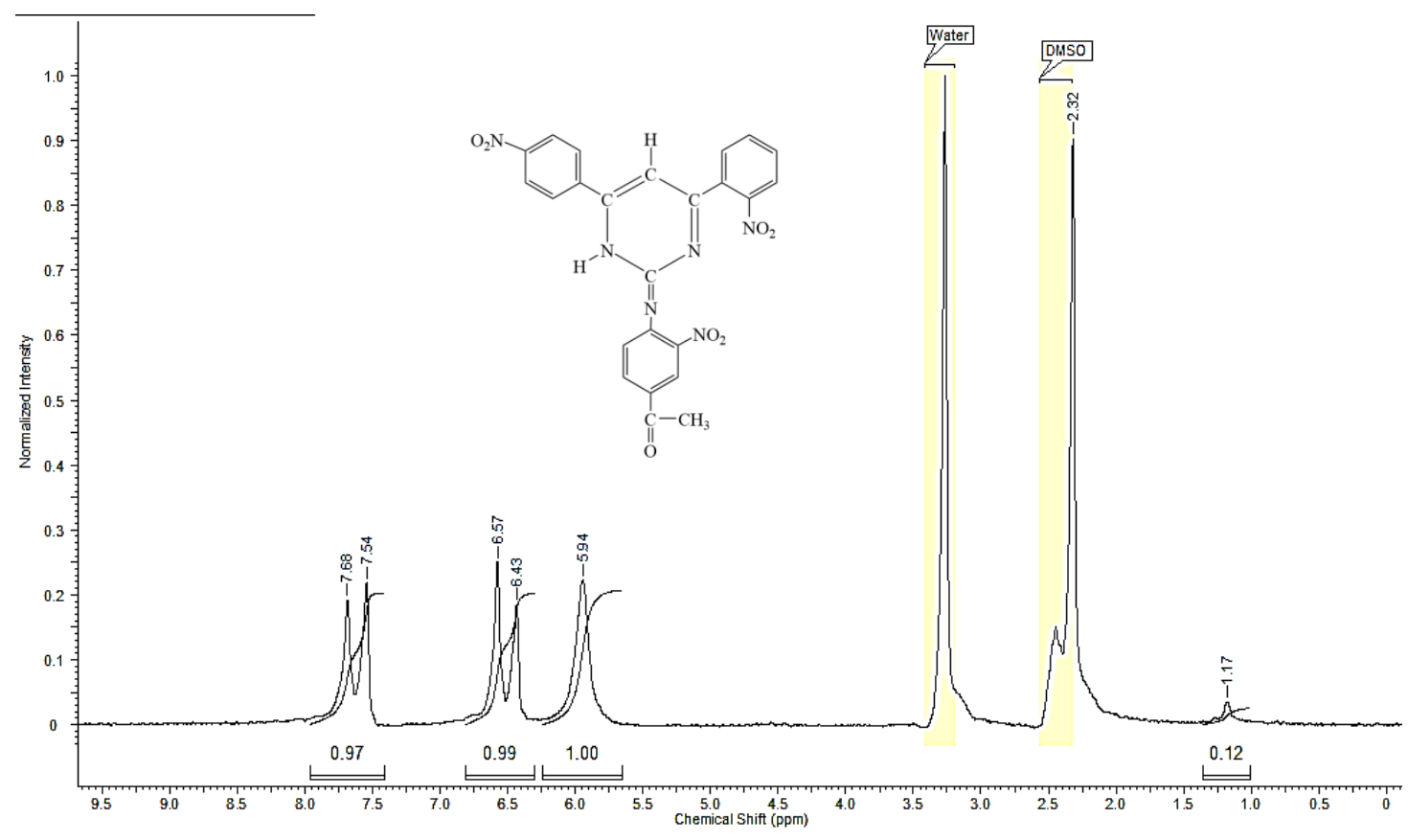

Fig (2): NMR spectrum of the compound (29) 


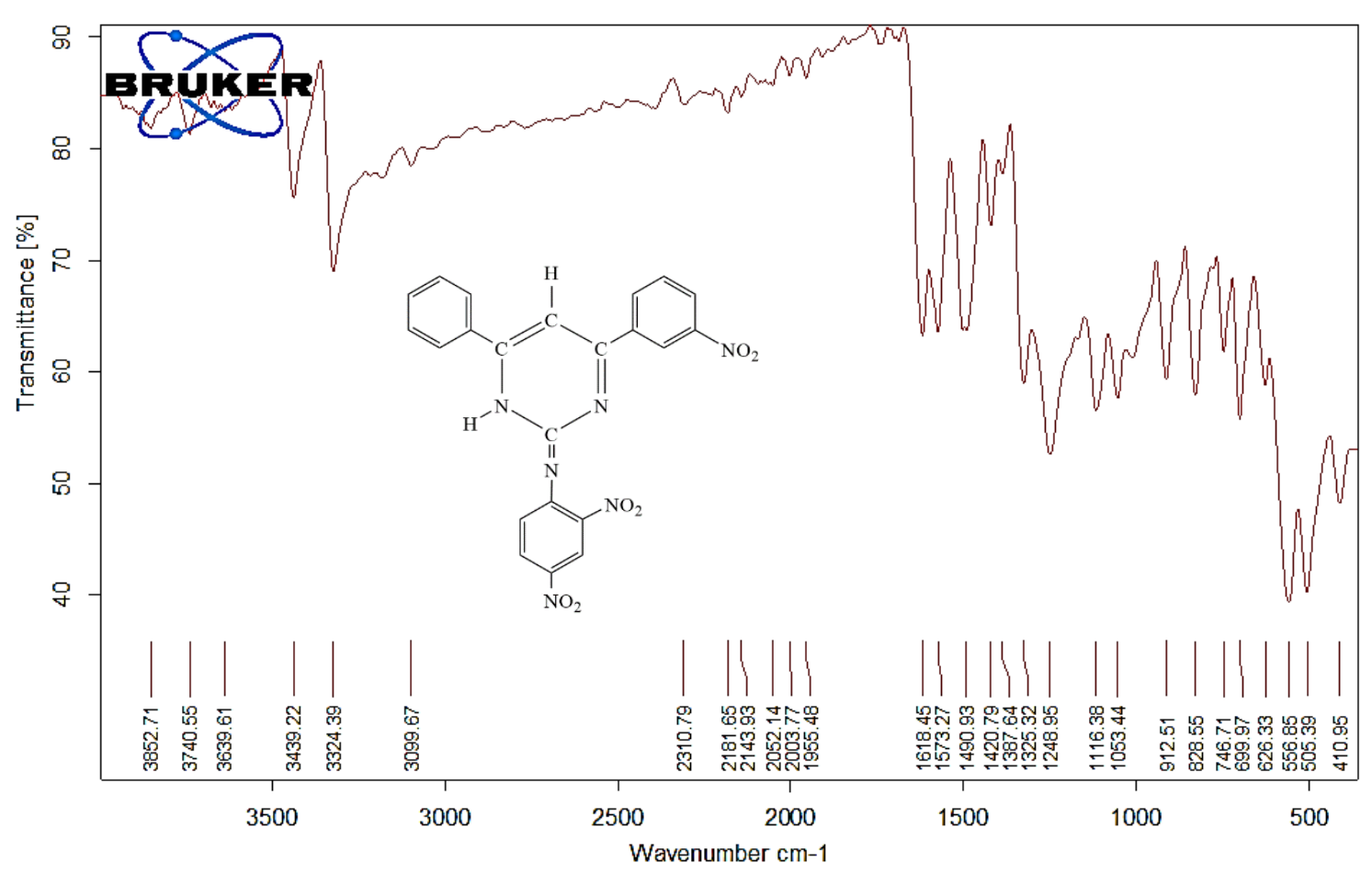

Fig (4): IR spectrum of the compound (35)

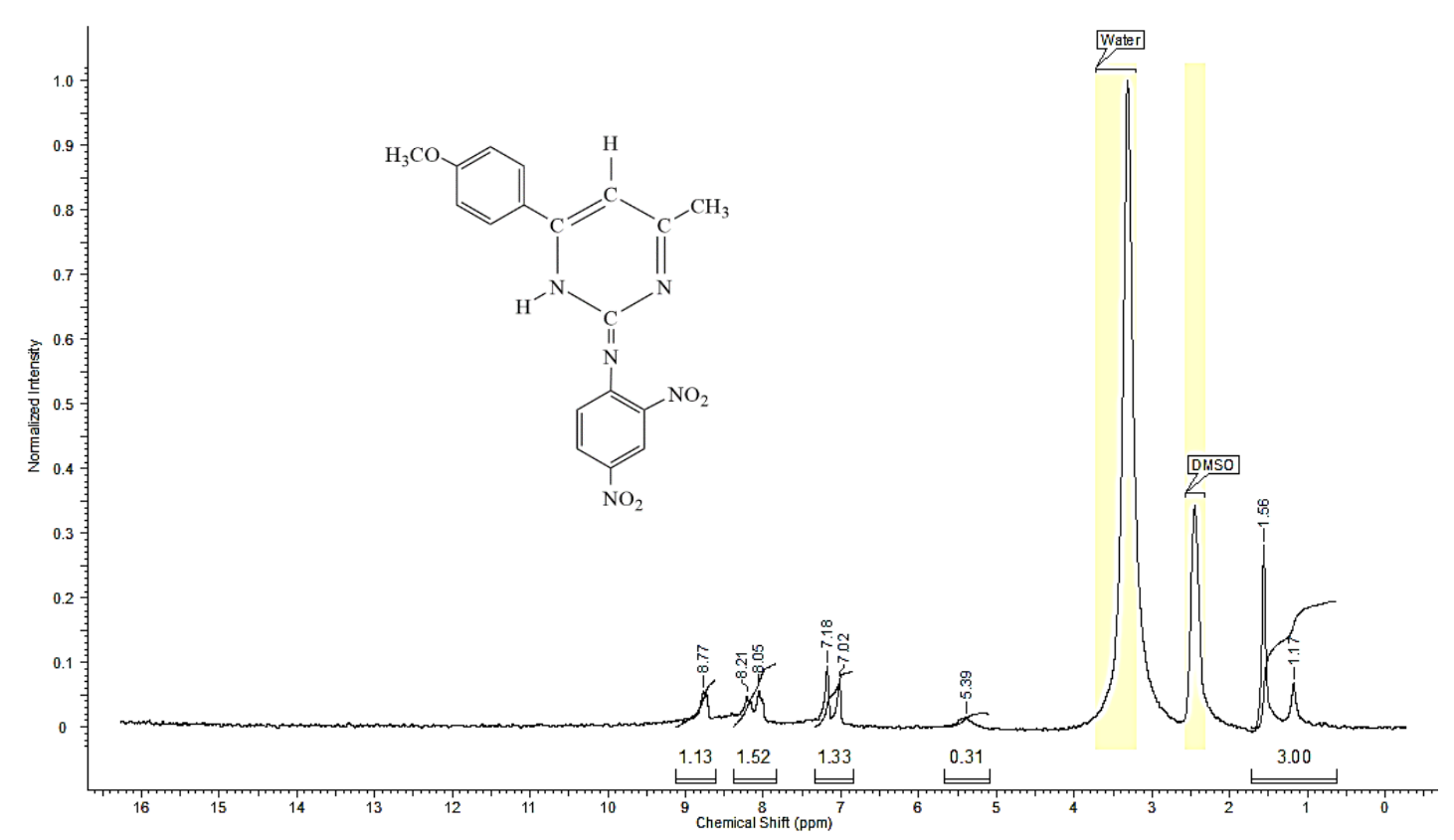

Fig (4): NMR spectrum of the compound (31) 


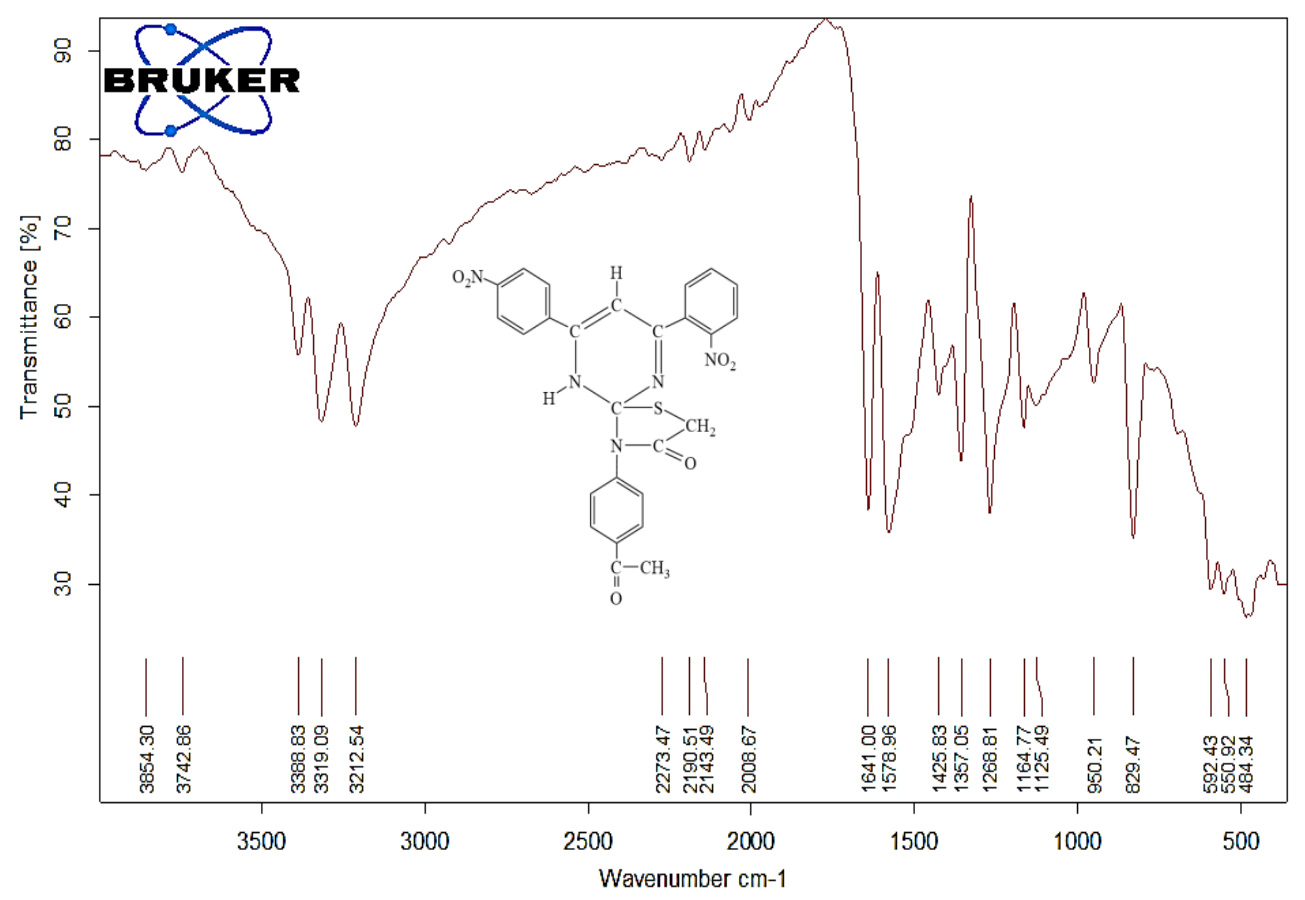

Fig (5): IR spectrum of the compound (49)

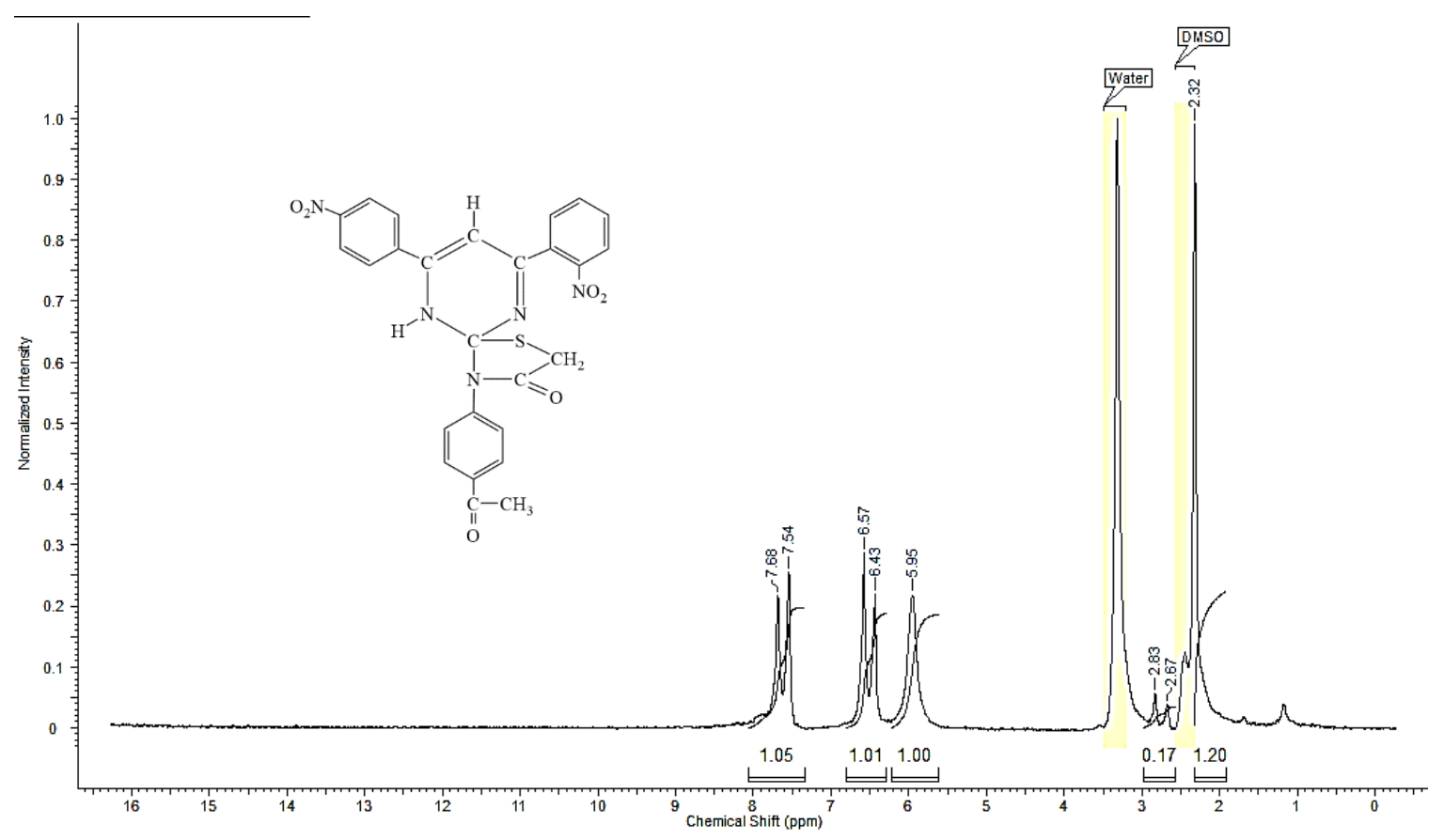

Fig (6): NMR spectrum of the compound (49) 


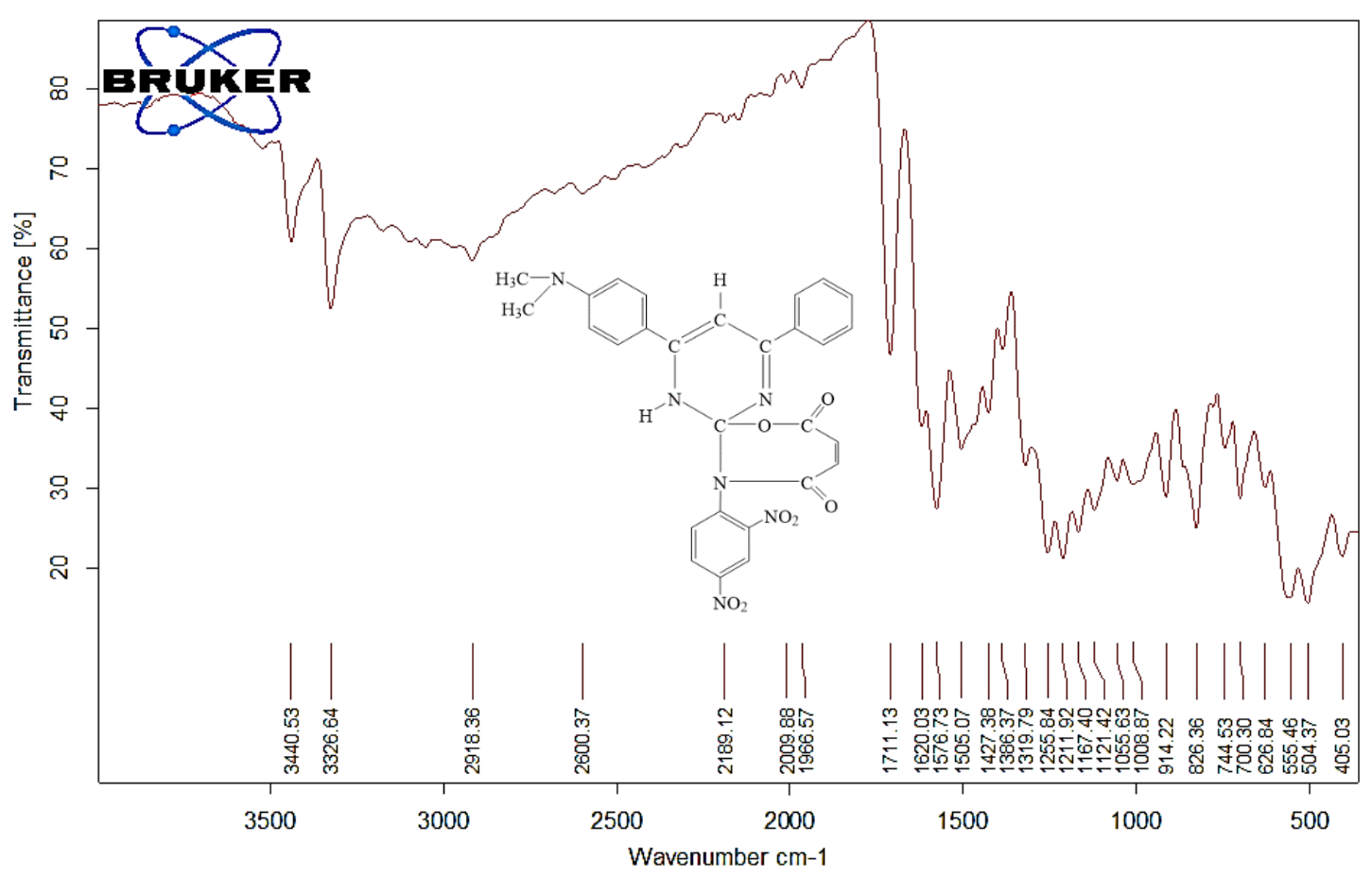

Fig (7): IR spectrum of the compound (54)

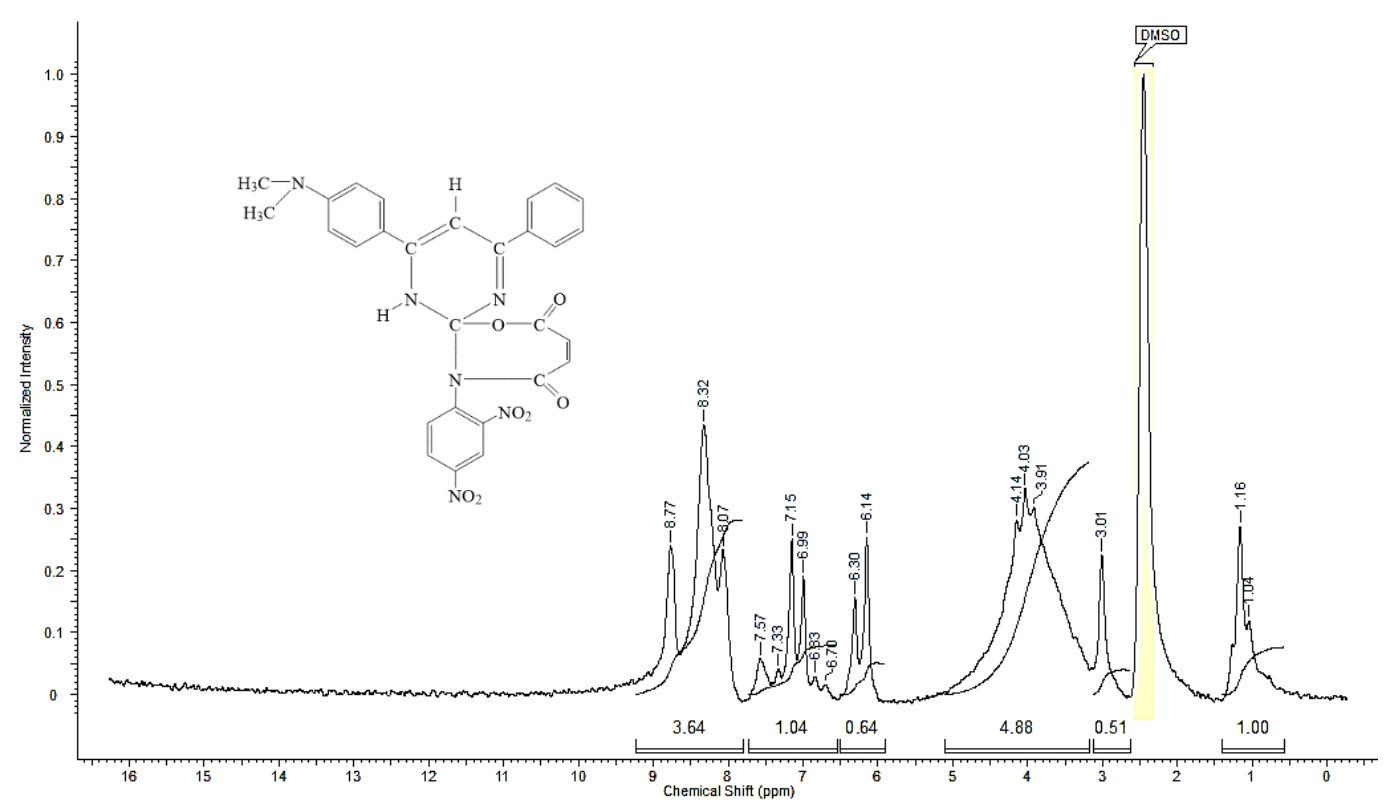

Fig (8): NMR spectrum of the compound (54) 


\section{REFERENCES}

1- karia F.D. ; Asian, J. Chem. 11, P.991-995, (1999).

2- Subudhi B.B., Tosh, B. k., Panda, P.K. Sahu, S. and Majhi, P., Dhaka Univ., J. Pharm. Sci, 4(2),8792. (2005).

3- Patel J. B. and Desai A., Int. J. Ind. Chem.,2,1, P.(45-51), (2011).

4- Srivustava S. k, Srivustava. S. and Srivustava S. D. ,Ind. J. Chem., 14 B, P. 1973-1945. (2002),

5- Parekh H. H., Parekh K. A., Parekh A. R., J. of Sci. Iran, 15,2, P.143-148. (2004).

6- Qien G. Li., Cui, X. Hueng, J. Q, D. Cui, Zhang R., Liu. F.,, J. of Fluorine Chem., 22, P. 182-186. (2006).

7- Aralmarugan S. k., Venkatraman H. P, , Rasayan. P. R. J. Chem., 3, 385, (2010).

8- Wadeher J. S., Puranik, P. M., Karande. A. N. Yeole. G. P., I. J. of Pharm. Tech. Research, P. 2233, (2009).

9- Desai S. B., Desai P. B., Hetrocyel Common7 (1), P.83-90, (2001).

10- Abdel Latif N. A., Saeed M. M., Ahmed N. S. ,Batrana R. Z. and El-Mouhty N. R., IJIRSC.Vol.3,Issuel 1,p.8517-8529,(2014).

11- Trived A. R., Dodiya D. K., Ravat N. R. and Shah V. H., ARKIVOC,P.131-141,(2008)

12- Ahmed A. A., Ph. D. Thesis.Department of chem, college of Education, University of mosul(2019).(In Arabic).

13- Mohammed J. M., Ahmed. Kh. A. Abachio, Tikrit J. of Pharm. Sci., 12(1), P.76-89, (2017).

14- Al-Rawi S. M., Ph. D. Thesis. Department of Chem, College of Education Ibn-Al Haitham, University of Baghdad. Baghdad, Iraq, (2012). (In Arbic).

15- Hussain Z., Yousif, E., Ahmed, A. and Altaie. A., Organic and Medicinal Chemistry Letters, Vol. 4, No.1, P1-4. (2014).

16- Lakum H. P., Shah, D. R. and Chikhalia, K. H., International Letters of Chemistry, Physics and Astronomy, Vol. 38, P. 56-73, (2014). 
17- AL- Mosawi S. K., Research Journal of Pharmaceutical Biological and Chemical Sciences, Vol. 5, No. 6, P. 411-417, (2014).

18- Hallinan A.E. Hagen J. T., Tsumbalov.s., Husa K. R., Stapelfeld. C.A., Savage. A.,39(2), M.,J. Med. Chem. P. 609.613, (1996).

19- Dhanya S., Ranjitha, C., Rama, M.; and Pai, K., International Journal of Innovative Research in Science Engineering and Technology, Vol. 3, Issue8, P.15357_15363, (2014).

20- Kumar k Chandrashekar K., Nagaraju ,G., and Nath, Li ; Vol. 4, No. 4, Pharma Chemica., (2012). Level A., 75s(9), Comm, Reg. P. 2187-2190., (2008). 